\title{
ROLE-PLAY SIMULATION OF TELEMEDICINE FOR UNDERGRADUATE HEALTH STUDENTS
}

\author{
Carrion B*, Portillo-Palma P and Mendez EA \\ Escuela de Medicina y Ciencias de la Salud, Tecnologico de Monterrey, Mexico
}

\begin{abstract}
Telemedicine has reemerged as the way to provide patient care amid the COVID-19 pandemic. It poses a safe and cost-efficient alternative to presence-based health appointments. Integrating remote patient care into healthcare training during their pre-clerkship curriculum is crucial for them to acquire professional skills to succeed in their postgraduate practices. One technique to achieve this is role-playing. It provides a learning environment for students to experience their future professional context in a safe, controlled setting. An educational innovation was implemented in the Preclinical Skills course to train students to carry out a teleconsult using peer role-play simulation. The study considered a mixed approach with a cross-sectional and descriptive design. The sample consisted of 75 students in the third semester on a health undergraduate program at Tecnologico de Monterrey organized in teams; each member represented a different role: health professional, patient, and observer-evaluator. Three clinical cases (abdominal pain, bad breath, and insomnia) randomly assigned. Each one with a script containing the patient's background, present illness with medical history. Students had to simulate a teleconsult according to their role, and the observer had to score a classmate's performance. To describe their experience, students completed an anonymous questionnaire. Our results showed that students identified key points that allow a teleconsult to successfully develop, even when not having the same role in the dynamic. They also recognized their opportunity areas and highlighted these tools as useful for obtaining skills that will help them excel in their professional practice even after the COVID-19 pandemic.
\end{abstract}

Keywords: emergency adaptation, remote teaching, educational innovation, role-play, simulation, telemedicine, health education, skills, role-play

\section{Introduction}

The COVID-19 pandemic is an unprecedented event that has reduced the opportunity to conduct inperson interaction, affecting all of our daily lives' aspects. Education is not an exception. Social distancing limits health students' exposure to patients jeopardizing learning opportunities and affecting clinical skill development. Challenging areas in the healthcare and educational system opens up. But while this might be discouraging, it is also an opportunity to implement new strategies and improve the healthcare system and teaching programs. It ought to seek innovative approaches that can be applicable alongside social distancing. Online simulation learning could help students develop professional skills.

The COVID-19 pandemic restricted exposure to patients and created the need for alternatives to faceto-face practices. Online simulation-based learning is a feasible strategy that can replace a certain extent of presenciality. This strategy promotes critical thinking (1) while providing a safe, controlled 
environment for undergraduate students to first approach a patient. Simulation can be started early in study programs since it works effectively for both freshmen and senior students (2).

The American Academy of Pediatrics (AAP, n.d.) emphasizes the importance of simulation as a technique that integrates cognitive, technical, and behavioral skills in a safe learning environment (3). Simulation-based learning has proved its efficiency by helping students to develop skills. It provides complex scenarios that could help them address the difficulty of real clinical cases (4). Figure 1 shows the potential of using role-play simulation to educate students, to reinforce and develop professional skills while using telemedicine.

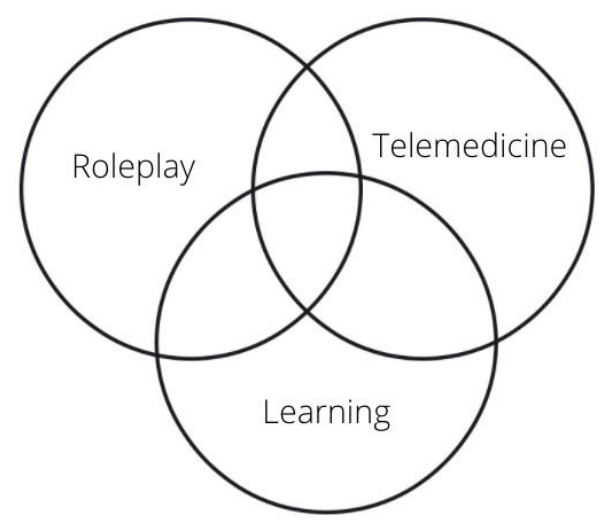

Figure 1: Interrelation between telemedicine and role-playing simulation to improve and develop the students learning skills

\section{Simulation in health sciences}

Simulation is accomplished in many ways. One of its aims is to develop collaborative skills since health approaches worldwide have shifted to an interdisciplinary team instead of an individual approach (5). Health students should communicate with peers and their patients hence, substantial communication capabilities are an essential professional attribute in modern health practice. Communication enhances patient empowerment and consequently increases healthy behaviors among them in short and long-term care (6). Critical thinking allows them to problem solving (diagnosis) and implementing accurate solutions (treatment) (2).

Regarding virtual and distance learning strategies, Role-playing is an exceptionally effective method to develop competences in health care, not only through active learning but also by improving the basic and clinical competencies of the health curriculum (1). It provides an opportunity for students to learn how to work in difficult circumstances to create a framework for future professional development (6). This simulation allows the educator to give feedback by analyzing the students' responses and reactions to the given situation, allowing them to obtain communication skills and to be an empathetic health care worker when they take the patient's role (6).

In the role-play, simulation-based training method, students can play different parts. In the SimP model (Simulated Patient), the patient's role is played by a patient, person, or actor (6), providing the student with a high degree of realism. On the other hand, the peer role-play (PRP) allows learners to 
play the patient and the health professional role, getting them to experience multiple perspectives and understand the complexity of the physician-patient interaction (7). Some authors have also explored the advantages of the PRP model compared to the SimP giving the PRP the advantage of being less expensive and having the same effectiveness as other simulation methods for teaching clinical skills in health students (6). PRP has also been shown to enhance the students' cognitive understanding and enrichen the affective experience (1). The PRP model is an excellent training tool in health education due to its low cost. It allows the students to develop the much-needed communication skills while also providing them with early introduction of clinical skills. These tools will help them improve their abilities to thrive interpersonal skills, enhancing confidence and comfort for future interaction with patients (8). These learning scenarios can also be useful to provide the students with an alternative to face-to-face practices amid the Covid-19 pandemic.

An adaptation of the PRP models was developed to give the students a practical approach to remote health care according to a standard teleconsult scheme, where students were assigned to a threemember team. One student played the Health Professional (HP) role, the second as the Patient (P) and the third one as the observer-evaluator $(\mathrm{O})$. Three different scripts were randomly assigned: abdominal pain, insomnia, and bad breath. The health professional had to question the patient (who also has a script provided by the teacher) about the reason for consultation and further instruct him/her to self-explore; the observer had to evaluate the classmates in their performance. Table 1 summarizes the role each student played in the interviews.

Table 1: Roles of the students during the Peer-role-play simulation of a teleconsult

\begin{tabular}{ll}
\hline Role & Activities \\
\hline Health professional & $\begin{array}{l}\text { Based on the assigned script with instructions conducts an } \\
\text { interview and guides self-exploration }\end{array}$ \\
\hline Patient & $\begin{array}{l}\text { Answers and delivers an open conversation about his/her chief } \\
\text { complaint and illness provided in the script }\end{array}$ \\
\hline Observer-Evaluator & $\begin{array}{l}\text { Observes and evaluates the dynamic based on the rubric of } \\
\text { interview and exploration given with the script }\end{array}$ \\
\hline
\end{tabular}

\section{Methods}

This study had a mixed approach, both quantitative and qualitative. It was conducted during the fall semester (August-December) of 2020. A remote adaptation in the course of Preclinical skills was made amid the Covid-19 pandemic. Students enrolled in this course were 3rd-semester undergraduate health students (Medicine, Dentistry, Nutrition, Biosciences, and Psychology). Seventy-five out of 200 students enrolled in the Preclinical skills course were included.

Students followed the instructions given by the teacher on the scripts of diverse clinical scenarios which included detailed instructions for each role and relevant clinical data with the health history. A video focused on self-exploration for each scenario was also given for students to watch before the teleconsult. Each script was assigned randomly. 
The teleconsult was recorded and submitted to the teacher for further revision. Along with the video, students completed an anonymous questionnaire to assess their learning experiences. All participants gave informed consent. The Ethics and Research Committee of Tecnologico de Monterrey approved the protocol, and it complied with the Declaration of Helsinki.

\section{Results}

The observer-evaluator had to assess their team members on their performance in a self-evaluation divided into an interview and guided self-exploration. Thirty-two self-evaluations were submitted: 18 regarding the interview process and 15 regarding the instructed self-exploration. Three teams were excluded from the self-exploration because the depression case did not require a physical exploration. The variables in the self-evaluation assessment were categorized on a scale from 1 to 3 (3 being the optimal expected performance). Table 2 shows the mean and variance of the students' self-evaluation scores. First on the interview, and then on the guided self-exploration.

Table 2: Performance evaluation

\begin{tabular}{lll}
\hline Interview & Mean & Variance \\
\hline Health professional introduction & 3 & 0 \\
\hline Introduction to patient & 3 & 0 \\
\hline Active listening & 2.8 & 0.11 \\
\hline Variability in the interview & 3 & 0 \\
\hline Content of the interview & 3 & 0 \\
\hline Empathetic communication & 2.93 & 0.06 \\
\hline Nonverbal communication & 3 & 0 \\
\hline Paraphrasing & 3 & 0 \\
\hline Guided self-exploration & & \\
\hline Hygienic measures & 2.66 & 0.26 \\
\hline Shares agenda to the patient & 3 & 0 \\
\hline Guided instruction provided & 3 & 0 \\
\hline Appropriate technique & 2.66 & 0.26 \\
\hline Palpation done after observation & 3 & 0 \\
\hline
\end{tabular}

A five-question survey was sent to the students focused on the role they played. From the 65 answered questionnaire form: 24 were regarding the role as Health Professional (HP), 21 as their role of a standardized-simulated Patient (P), and 20 were as their role of the observer-evaluator (O). These surveys consisted of four items with multiple-choice questions and one open-ended question. The open-ended question inquired about the student's learning experience in the peer role-playing, categorizing the answers into responsibility, communication, empathy, and role-playing relevance.

Noteworthy quotes provided by the students' perception of the role-played either as a Health Professional (HP), the Patient $(\mathrm{P})$, and the Observer $(\mathrm{O})$ are listed below: 


\section{Responsibility}

Students acknowledged the importance of interaction between the health professional and the patient to achieve successful consultation.

- I understood how important it is to avoid health terms while interviewing the patient. (HP)

- In role-play, adopting the role of the doctor provides a teaching exercise. (HP)

- The importance of helpful consultations depends equally on the health professional as the patient (P)

- The role-play dynamic offered the possibility to understand how the patient feels and must conduct in a consultation $(\mathrm{P})$

The observer recognized that small details influence the interaction between the health professional and the patient.

- I was able to identify how small details such as the background, visual contact, and appropriate clothes can provide a comfortable atmosphere for the patient. (O)

\section{Communication}

Students recognized the importance of communication between the health professional and patient and its impact on a successful consultation.

- I realized how hard it is to communicate bad news. (HP)

- I acknowledged the importance of asking appropriate questions to obtain useful information for diagnosis. (HP)

- I realized that non-verbal communication is as important as the verbal one. (HP)

- As a patient, the details I share about my disease are equally relevant for diagnosis, as the health professional questions. (P)

- I understood the importance of listening to health recommendations in a nonjudgmental manner. $(\mathrm{P})$

Observers noted that communication on a teleconsult is a two-way process.

- I was able to analyze the different roles and how to communicate appropriately. (O)

\section{Empathy}

Students acknowledged the importance of a safe environment for optimal consultation.

- Overcoming social distance in consultation is obtained by providing a safe and comfortable atmosphere. (HP)

- As a health professional in teleconsult, one should be patient when guiding selfexploration. (HP)

- Empathy is bidirectional between health professionals and patients. $(\mathrm{P})$

- I could understand how important it is to feel comfortable and being listened to. (P)

Observers also noted the importance of a professional attitude on a healthcare professional. 
- As an observer, one must remain neutral. (O)

- I recognized how necessary professionalism provides seriousness to the teleconsult. (O)

\section{Relevance of role-playing}

Students recognized the importance of being prepared for a teleconsult, the benefits, and challenges of receiving feedback from a tutor.

- There is a difference between learning about diseases and interviewing a sick patient. (HP)

- I rehearsed the appropriate way to do an interview and guide self-exploration applied to the actual reality of COVID-19. (HP)

- Tutor feedback allowed me to understand the reason behind the scenes of interviewing formally and understandably (HP)

- I could understand how challenging the teleconsult can be without physical contact. $(\mathrm{P})$

- I understood the importance of building a relationship based on trust (P)

Observers acknowledged their part in the dynamic even when being in a passive role and could benefit from observing the dynamic of a teleconsult.

- Even though I did not have an active role, I participated in the preparedness of the dynamic. (O)

- I could visualize how the health professional and patient conduct during a consultation. (O)

- Identify mistakes and assets during the health consultation of my team members. $(\mathrm{O})$

\section{Discussion}

Innovation and adaptation into educational programs must keep up with a rapidly changing world. Online learning tools such as role-playing or simulation-based learning helps online teaching in the health program curriculum, allowing students to achieve relevant clinical skills needed in their future practices in the post-pandemic time (9). We need to move forward and use technological resources to our advantage, looking for innovative ways to improve patient care, starting with the way we teach undergraduate health students.

Some studies have focused on the importance of students training on telemedicine $(10,11,12)$ which plays an important role in healthcare delivery and management of patients in the Covid-19 pandemia since it allows communication with any available technological device, avoiding physical contact, and limiting exposure as reducing the risk of virus infection $(9,11)$.

This study offered many insights on the importance of simulation in student development. The selfevaluation provided students' awareness on opportunity areas to work in specific skills. Results showed that the lowest scored items were active listening, empathetic communication, and instruction to use proper hygienic measures. These give cues for students to not only identify but also to address them. One of the main challenges in professional training can be to achieve successful crosstalk with the patient. Students' low scores on active listening may be due to angst related to allowing an open conversation can cause missing valuable clinical information during a consult. Efficient 
communication skills in health professionals break down the barriers between them and the patient, redirecting the interview to fill in gaps to achieve an accurate diagnosis (13).

Another cardinal key to patient-centered communication is empathy towards the patient by understanding, respecting, and supporting him/her (13). An inexperienced professional may be anxious about conducting the interview and narrowing the information to achieve a diagnosis and establishing a plan. Overall, this might distract them and forget to remain empathetic with the patient. These could explain the low score on empathy which can be acceptable and expected since many students encountered their first patient with role-playing.

Another item where the students scored themselves low was the usage of hygienic measures. It is stressed that hand hygiene is the responsibility of all staff members in a hospital. Epidemiological evidence for hospital infections associated with non-compliance with hand hygiene represents $8-12 \%$ of patients' infections admitted to hospitals (14). The low score in hygienic measures in our findings suggests that it should be emphasized to students that the first step in any exploration is a proper hand hygiene technique with either soap or a disinfectant gel.

Interestingly, on the learning experience, all team members arrived at similar observations during the role-play and managed to identify important details that could help them achieve a successful interaction with a patient, even when not having the same role. The experience became a challenge for health students who had to play roles they had not done before. Nevertheless, this acts on behalf of molding a better healthcare professional by analyzing different perspectives and understanding the importance of empathy towards their patients. Health students could reflect on the importance of a thorough and straightforward conversation with an open and honest interview both from the health professional and patient, which sets up a scenario to direct a proper diagnosis and management. In teleconsult, they acknowledged that the professional attire and visual background sets up the atmosphere to be professional and confident. They also agreed that this first introduction to patient care by remote communication sets the basis for Telemedicine, which could become a standard of care in the future.

\section{Conclusion}

Early student exposure to patients has proven to be an effective tool in health professional formation. Simulation has proven to be an excellent tool, however, some limitations such as economic resources and facilities make it not available for everyone.

Adaptations to simulation models, especially peer role-play, can provide a low-cost, risk-free, and safe setting to expose students to patients early on their formation. It helps them achieve relevant skills for healthcare professionals, which should be acquired throughout their education. Role-playing simulations create the perfect setting to favor their growth. This study proposes the introduction, early in the health programs curriculum, of simulation dynamics such as peer-role-playing to train and allow students to rehearse and develop several skills needed to thrive in their professional practice. Future implementations should focus on the students becoming proficient in soft skills using remote simulation to develop them. This will train future professionals that care for patients in an integral view. . 


\section{Acknowledgements}

The authors would like to acknowledge the financial support of Writing Lab, TecLabs, Tecnologico de Monterrey, Mexico, in the production of this work.

\section{References}

1.Manzoor, I. Mukhtar, F., Hashmi, N. (2012). Medical Students' Perspective About Role-Plays As A Teaching Strategy in Community Medicine. Journal of the College of Physicians and Surgeons Pakistan, 22(4), 222-225.

2.Chernikova, O., Heitzmann, N., Stadler, M., Holzberger, D., Seidel, T., \& Fischer, F. (2020). Simulation-Based Learning in Higher Education: A Meta-Analysis. Review of Educational Research, 90(4), 499-541.

3.American Academy of Pediatrics. (n.d.). Simulation Based Learning. Retrieved 1 February 2021, from https://www.aap.org/en-us/continuing-medical-education/life-support/Pediatric-Education-forPrehospital-Professionals/Pages/Simulation-Based-Learning.aspx

4.Knowles, C., Kinchington, F., Erwin, J., \& Peters, B. (2001). A randomised controlled trial of the effectiveness of combining video role-play with traditional methods of delivering undergraduate health education. Sexually transmitted infections, 77(5), 376-380.

5.Epstein N. E. (2014). Multidisciplinary in-hospital teams improve patient outcomes: A review. Surgical neurology international, 5(Suppl 7), S295-S303.

6.Gelis, A., Cervello, S., Rey, R., Llorca, G., Lambert, P., Franck, N., Rolland, B. (2020). Peer RolePlay for Training Communication Skills in Medical Students. Simulation in Healthcare: The Journal of the Society for Simulation in Healthcare, 15(2), 106-111.

7.Bosse, H. M., Nickel, M., Huwendiek, S., Jünger, J., Schultz, J. H., Nikendei, C. (2010). Peer roleplay and standardised patients in communication training: a comparative study on the student perspective on acceptability, realism, and perceived effect. BMC health Education, 10(1).

8.McIvor, L., Karnes, M. (2019). Role-Play as an Effective Way to Teach Relationship Building with Telehealth. The Open Journal of Occupational Therapy, 7(2).

9.Hollander, J. E., Carr, B. G. (2020). Virtually Perfect? Telemedicine for Covid-19. New England Journal of Medicine, 382(18), 1679-1681.

10.Waseh S, Dicker AP. (2019). Telemedicine Training in Undergraduate Medical Education: MixedMethods Review. JMIR Medical Education, 5(1), e12515.

11.Martinez L, Holley A, Brown S, Abid A. (2020). Addressing the Rapidly Increasing Need for Telemedicine Education for Future Physicians. PRiMER (Leawood, Kan.), 4, 16.

12.Doğantan Ece, (2020). An interactive instruction model design with role play technique in distance education: A case study in open education system. Journal of Hospitality, Leisure, Sport \& Tourism Education, 27, 100268.

13.Hashim M. J. (2017). Patient-Centered Communication: Basic Skills. American family physician, 95(1), 29-34.

14.Alifragis. D., Alikari, V. and Kelesi. M. (2019). The importance of hand hygiene in healthcare settings. International Journal of Midwifery and Nursing Practice, 2(1), 102-105. 